\title{
Structural Genomics-A Goldmine of Blueprints for Structure-Based Drug Design
}

\section{Garry W. Buchko}

Biological Sciences Division, Pacific Northwest National Laboratory, Richland, WA

Multidrug resistant bacterial pathogens pose a serious threat to modern medicine, threatening to make some common medical procedures obsolete [1] and the treatment of some common bacterial infections difficult [2]. For example, while effective public health care systems in the Western world keep Mycobacterium tuberculosis, the aetiological agent responsible for tuberculosis, under control, the evolution of multidrug and extremely drug resistant strains of M. tuberculosis may result in the sudden loss of this control [3]. Consequently, it is of fundamental importance to develop a new generation of intervention strategies against bacterial pathogens.

One strategy to develop the next generation of antimicrobial agents is structure-based drug design, an approach that is playing a growing role in new drug discovery $[4,5]$. This method requires the acquisition of a three-dimensional structure of the protein obtained from X-ray diffraction (XRD) or nuclear magnetic resonance (NMR) methods. These methods for structure determination have advanced substantially over the past few decades due, in part, to easier access to synchrotrons and the high-throughput requirements of the NIH funded Protein Structure Initiatives I and II. At the same time the genomes of most pathogenic organisms have been sequenced. This "perfect storm" of technologies and genome sequences has resulted in the creation of structural genomic consortia dedicated to determining the structures of potential protein drug targets from infectious disease organisms. These proteins structures are the blueprints for the structure-based design of the next generation of drugs against bacterial pathogens and other infectious diseases.

In late 2007 the National Institute of Allergy and Infectious Diseases (NIAID) funded two consortia to determine protein structures from biodefense organisms and emerging and re-emerging infectious disease organisms: The Seattle Structural Genomics Center for Infectious Diseases (SSGCID;http://www.ssgcid.org)[6] and the Center for Structural Genomics of Infectious Diseases (CSGID; http:// www.csgid.org) [7].

The organisms include 31 different genera of prokaryotes, eukaryotes, and viruses from NIAID Category A, B, and C pathogens. Both centers actively solicit the infectious disease research community to identify essential enzymes, virulence factors, drug targets, and vaccine candidates of biomedical relevance and freely provide for them structural data. Indeed, the structural data is provided to the entire scientific community as all structures are immediately deposited into the RCSB Protein Data Bank (PDB; http://rcsb.org) and released. As of August 16, 2011, over 740 protein structures have been deposited into the PDB by both centers and it is projected that they will deposit over 1000 structures upon completion of their five-year mandate, a goldmine of blueprints for structure-based drug design.

In an effort to advertise the wealth of information available, an entire volume of Acta Crystallographica Section F has been devoted to the research efforts of the SSGCID [8]. The 29 research articles in the September 2011 issue includes an overview [8], seven laboratory communications on the details of the high-throughput pipeline, and 21 structural communications describing protein structures determined using XRD and NMR methods. In total, the 21 structural communications describe $41 \mathrm{PDB}$ entries of proteins from 14 different infectious disease organisms including M. tuberculosis (TB), Brucella melitensis (brucellosis), Giardia lamblia (giardiasis), Rickettsia prowazekii (typhus), and Bartonella henselae (cat scratch fever). Readers of the Metabolomics are encouraged to view this special issue of Acta Crystallographica Section F to obtain an idea of the unique structural genomics resources freely available to assist in the discovery and development of novel cures or treatments for infectious diseases.

\section{References}

1. World Health Organization (2000) Overcoming antibiotic resistance. World Health Organization, Geneva.

2. Bertino JS Jr (2009) Impact of antibiotic resistance in the management of ocular infections: the role of current and future antibiotics. Clin Ophthalmol 3: 507-521.

3. Russell DG, Barry III CE, Flynn JL (2010) Tuberculosis: What we don't know can, and does, hurt us. Science 328: 852-856.

4. Mueller L, Montelione G (2002) Structural genomics in pharmaceutical design $\mathrm{J}$ Struct Func Genomics 2: 67-70.

5. Van Voorhis WC, Hol WG, Myler PJ, Stewart LJ (2009) The role of medical structural genomics in discovering new drugs for infectious diseases. Plos Comput Biol 5: e1000530.

6. Myler PJ, Stacy R, Stewart LJ, Staker BL, Van Voorhis WC, et al. (2009) Seattle Structural Genomics Center for Infectious Disease (SSGCID). Infect Disord Drug Targets 9: 493-506.

7. Anderson WF (2009) Structural genomics and drug discovery for infectious diseases. Infect Disord Drug Targets 9: 507-517.

8. Stacy R, Begley DW, Phan I, Van Voorhis WC, Varani G, et al. (2011) Structura genomics of infectious disease drug targets: the SSGCID. Acta Crystallogr $F$ 67:979-984.

Corresponding author: Garry W. Buchko - Biological Sciences Division, Pacific Northwest National Laboratory, Richland, WA 99352, USA, Tel: 509-371-6543; E-mail: garry.buchko@pnnl.gov

Received August 22, 2011; Accepted October 22, 2011; Published October 24 2011

Citation: Buchko GW (2011) Structural Genomics-A Goldmine of Blueprints for Structure-Based Drug Design. Metabolomics 1:104e. doi:10.4172/21530769.1000104e

Copyright: (c) 2011 Battelle Memorial Institute. This is an open-access article distributed under the terms of the Creative Commons Attribution License, which permits unrestricted use, distribution, and reproduction in any medium, provided the original author and source are credited. 